\title{
ASSOCIAÇÕES DE MOLUSCOS DO INFRALITORAL DE SANTA CATARINA, SC - BRASIL
}

\author{
ALMEIDA, T. C. M.; ROHR, T. E. \& C. SCHIO
}

\author{
Centro de Educação Superior de Ciências Tecnológicas da Terra e do Mar - CTTMar/ \\ UNIVALI, C.P. 360, CEP 88302-202, Itajaí, SC \\ e-mail: tito@univali.br
}

\begin{abstract}
RESUMO
Padrões de associações entre espécies de moluscos podem fornecer boas indicações ecológicas do ecossistema costeiro. O infralitoral centro-norte de Santa Catarina, compreendido entre Gravatá e a llha de Porto Belo, foi amostrado entre setembro de 2001 e dezembro de 2002 (8 locais entre Gravatá e Laranjeiras e 4 na llha). As amostras foram obtidas com auxílio de um busca fundo tipo van-veen com $0,025 \mathrm{~m}^{2}$ de área amostral, para os locais com profundidades entre 10 e 15 metros (de Gravatá até Laranjeiras), e através de mergulho autônomo utilizando tubos de PVC com áreas de $0,008 \mathrm{~m}^{2}$ e $0,018 \mathrm{~m}^{2}$, em quatro locais ao redor da llha de Porto Belo, com profundidades variando de 3 a 6 metros. Foram coletadas em cada local pelo menos 5 amostras que foram lavadas em peneiras com $0,5 \mathrm{~mm}$ de abertura de malha. O material retido nas peneiras foi fixado em solução de formol a $4 \%$ e transferido para solução de álcool a 70\% após a separação do sedimento retido nas peneiras. Amostras adicionais em todos os locais foram coletadas para a caracterização granulométrica. A similaridade entre os locais amostrados foi calculada por meio do coeficiente de Jaccard. Sobre a matriz de similaridade foi aplicada a análise de agrupamento utilizando o método das médias aritméticas não ponderadas na formação dos grupos. A análise de correspondência foi utilizada na identificação das associações entre as espécies e os locais, tendo sido as espécies com apenas uma única ocorrência excluídas das análises. Foi identificado um total de 42 taxa sendo: 1 Polyplacophora, 7 Gastropoda e 34 Pelecypoda. Os moluscos mais freqüentes foram Tellina spp., Natica lívida e Abra sp. Foram identificadas duas associações de espécies, uma que caracterizou as estações entre Gravatá e Laranjeiras; e outra a llha de Porto Belo
\end{abstract}

Palavras-chave: Associação, Moluscos, Infralitoral, Santa Catarina.

\section{SUBTIDAL MOLLUSK ASSEMBLAGE OF SANTA CATARINA COAST, SC - BRAZIL}

\begin{abstract}
Mollusk association patterns can provide good ecological indications in coastal ecosystems. The subtidal marine environment from Gravatá to Porto Belo Island in Santa Catarina state/Brazil, was sampled from September 2001 to August 2002. Sediment was sampled in this area with a van-veen grab $\left(0,025 \mathrm{~m}^{2}\right.$ of sampling area) at 10 to 12 meter depths (Gravatá to Laranjeiras). At Porto Belo Island samples were taken by scuba diving with one core that sampled an area of 0,018 $\mathrm{m}^{2}$. At least five samples were collected in each station. Samples were washed in 0,5 mm mesh size sieves and fixed with $10 \%$ formalin. Similarities between samples were calculated by Jaccard coefficient. Over the similarity matrix cluster analysis were applied using the UPGMA method to form the groups. Correspondence analysis was applied to identify the mollusk association. Forty two benthic taxa were identified: 1 Polyplacophora, 7 Gastropoda and 34 Pelecypoda. Most frequent mollusks were Tellina spp., Natica livida and Abra sp. Two assemblages were identified:
\end{abstract}


ALMEIDA et al:: Associações de moluscos do infralitoral de Santa Catarina.

one encompassed the area between Gravatá to Laranjeira and the other characteristic of the Porto Belo Island.

Key-words: Assemblage, Mollusks, Subtidal, Santa Catarina state.

\section{INTRODUÇÃO}

Os padrões de associação entre espécies fornecem excelentes informações sobre o ambiente marinho, principalmente quando se trata do fundo sedimentar. Os organismos bênticos se destacam no aspecto mencionado por apresentarem íntima relação com o substrato e possuirem padrões alimentares bem definidos. Muitas das espécies já foram estudadas e informações sobre produção e crescimento estão disponíveis para a ciência; integram diversas cadeias alimentares bentopelágicas e apresentam padrões espaciais e temporais bem definidos.

Os moluscos constituem um importante grupo faunístico para o bentos em geral, ocorrendo em muitos ambientes sedimentares de forma expressiva tanto em termos de abundância quanto em riqueza de espécies. Fazem parte da dieta de muitos vertebrados e invertebrados, desempenhando um importante papel ecológico.

As espécies de moluscos da costa brasileira são relativamente bem conhecidas como pode ser verificado através de Rios (1994). A maioria dos trabalhos realizados no pais apresentam listas de espécies excelentes que possibilitam um bom conhecimento acerca dos padrões de distribuição destas espécies, principalmente na costa centro sul do Brasil (Absalão, 1989; Absalão e Cruz, 1990; Migotto et al. 1993).

Uma abordagem mais ecológica em relação a este grupo faunístico foi dada por Absalão (1986); Gonçalves \& Lana (1991) e Arruda \& Amaral (2003), relacionando associações de espécies a variáveis ambientais tais como a salinidade e a granulometria.

O presente estudo teve como objetivo contribuir para o conhecimento da biodiversidade malacológica, bem como identificar as associações de espécies no infralitoral centro-norte de Santa Catarina.

\section{Área de estudo}

O estudo foi realizado na costa centronorte do Estado de Santa Catarina, nas imediações dos municípios de Navegantes, Itajaí, Balneário Camboriú e a llha João da Cunha no município de Porto Belo (Fig. 1). O aporte continental representado pelo deságüe do rio ItajaíAçú, no litoral de Itajaí e Navegantes, bem como outros rios de menor porte, exercem grande influência na plataforma interna (Schettini \& Carvalho, 1998). O rio Itajaí-Açú apresenta alto grau de comprometimento ambiental, decorrente das constantes dragagens do canal de acesso ao Porto de Itajaí, bem como pelo aporte de efluente urbano e industrial de toda a bacia hidrográfica.

A llha João da Cunha, popularmente conhecida como llha de Porto Belo, está situada a cerca de 900 metros da costa, sendo muito explorada pelo turismo, principalmente no período de verão. Em alguns locais da ilha pequenos cultivos de mexilhões são encontrados, bem como um elevado trânsito de embarcações, que aumentam no período de verão.

\section{MATERIAIS E MÉTODOS}

\section{Coleta de dados}

Foram realizadas amostragens mensais durante um ano, no período de setembro de 2001 a agosto de 2002, em 8 estações situadas nas praias de: Gravatá, Navegantes, Atalaia, Brava, Barra Norte, Barra Sul, Laranjeiras e no rio Itajaí-Açú, com profundidades entre 10 
e 15 metros. Foram coletadas 6 amostras por estação, reunidas duas a duas, perfazendo um total de 3 unidades amostrais, utilizando para isto um busca-fundo do tipo van-veen com $0,025 \mathrm{~m}^{2}$ de área amostral. No município de Porto Belo, foram amostrados 4 pontos ao redor da ilha, os quais possuíam de 3 a 6 metros de profundidade: Maricultura, Trapiche, Trilha 3 e Trilha 4 (Fig. 1), nos meses de julho, agosto e setembro de 2002. As coletas de sedimento foram realizadas por meio de mergulho autônomo, utilizando cilíndros de PVC com 10 e $15 \mathrm{~cm}$ de diâmetro, que perfazem respectivamente 0.008 e $0,018 \mathrm{~m}^{2}$, enterrados a uma profundidade de $15 \mathrm{~cm}$ no sedimento, sendo coletadas 5 amostras por estação em cada mês. Amostras adicionais de sedimento foram coletadas em diferentes épocas do ano nas
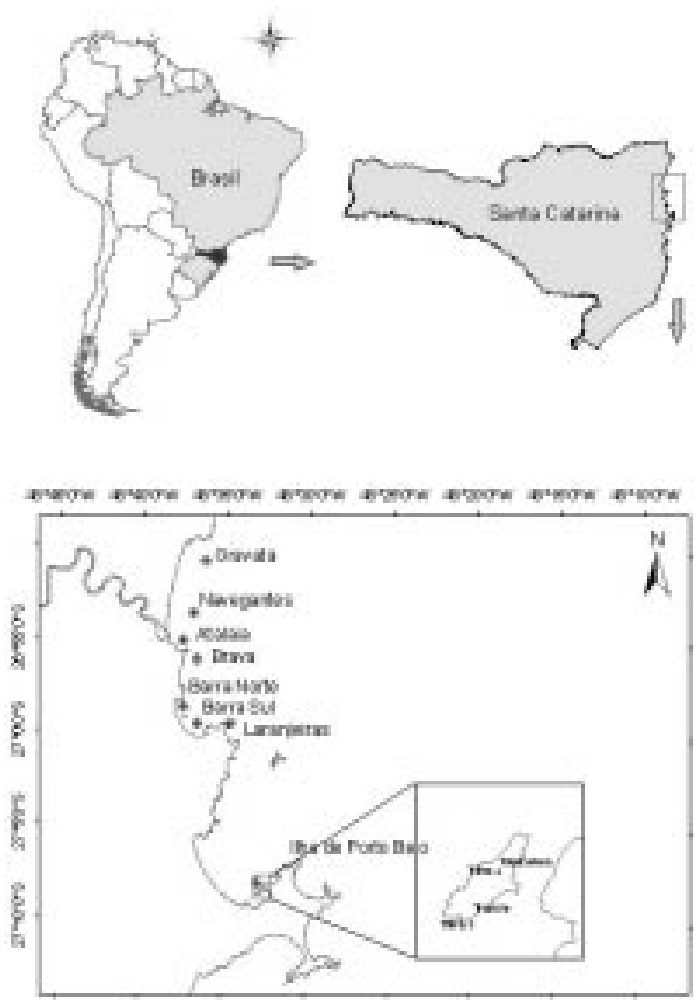

Figura 1 - Localização da Área de Estudo, compreendida desde Gravatá a Porto Belo. estações entre Gravatá e Laranjeiras, e em todas as amostragens realizadas na llha de Porto Belo, para a caracterização do tamanho médio do grão e do grau de seleção.

\section{Procedimento de campo e laboratório}

Em campo, cada amostra foi acondicionada em sacos com $0,5 \mathrm{~mm}$ de abertura de malha para a separação da macrofauna, sendo posteriormente fixada em solução salina de formol a $4 \%$. No laboratório, o material foi separado em grandes grupos com auxílio de microscópio estereoscópico e transferido para solução de álcool a $70 \%$, sendo posteriormente os moluscos identificados segundo Rios (1994). Para as análises posteriores foram considerados somente os espécimes com parte mole ou quando a concha apresentava perióstraco.

As análises granulométricas foram realizadas por peneiramento para os grosseiros e pipetagem para os finos de acordo com os procedimentos descritos por Suguio (1979) e o tamanho médio do grão e o grau de selecionamento estimados segundo Folk \& Ward (1957).

\section{Análise dos Resultados}

Para análise das associações foram consideradas somente a presença e a ausência das espécies e, com base nessas, calculada a freqüência de ocorrência absoluta e relativa aos locais amostrados. A similaridade faunística entre os locais foi calculada através do coeficiente de Jaccard e sobre a matriz de similaridade foi aplicado o método das médias aritméticas não ponderadas para a identificação das associações. Ainda sobre os dados de presença e ausência foi aplicada uma análise de correspondência para a identificação das associações entre as espécies (Legendre \& Legendre, 1998). Para todas as análises aplicadas na identificação das associações foram excluídas as espécies com apenas uma ocorrência. 


\section{RESULTADOS}

\section{Granulométricos}

A região compreendida entre Gravatá (limite norte da área amostrada) e Laranjeiras apresentou sedimentos síltico argilosos mal selecionados, enquanto que o sedimento no entorno da llha de Porto Belo (limite sul da área amostrada) foi caracterizado por frações mais grosseiras com melhor grau de selecionamento (Fig. 2).

\section{Malacológicos}

Foram identificados 42 taxa pertencentes ao filo Mollusca, sendo 32 identificados ao nível de espécie e 10 ao nível de gênero e/ou família. Os taxa mais freqüentes foram respectivamente Tellina spp, Natica cf. lívida e Abra $\mathrm{sp}$ (Tab. 1).

Foi possível identificar duas grandes associações entre os locais amostrados (Fig. 3), uma constituída pelas estações ao longo da costa entre Gravatá e Laranjeiras e outra ao redor da llha de Porto Belo. Em ambas as associações foi possível identificar agrupamentos menores entre os locais. Na ilha foi possí-

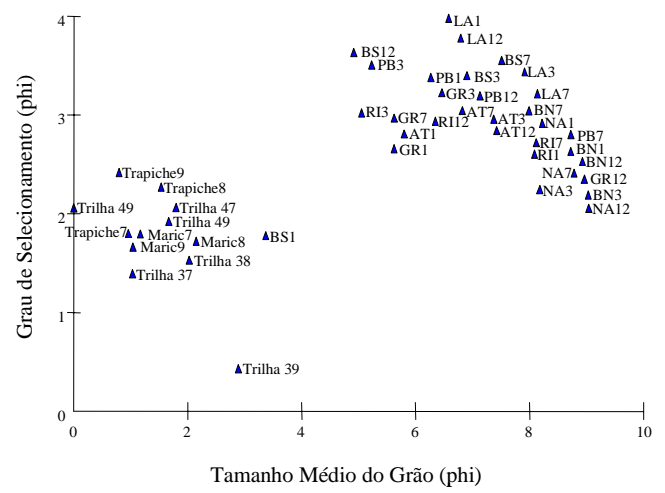

Figura 2 - Tamanho médio do grão e grau de selecionamento (phi) do fundo sedimentar dos locais amostrados. Locais representados por siglas representam as estações entre Gravatá e Laranjeiras e as palavras representam os locais no entorno da ilha de Porto Belo. vel identificar dois grupos menores, sendo um constituído pela área representada pelo trapiche de acesso à ilha e a área de cultivo de moluscos (Trapiche e Maricultura), e a outra reuniu os locais representados pelas Trilhas 3 e $4 . \mathrm{Na}$ outra grande associação foi verificado o isolamento da estação posicionada dentro do Rio Itajaí.

A análise de correspondência evidenciou ao longo do 10 eixo fatorial o mesmo padrão de associação encontrado através da análise de agrupamento (Fig. 4). A distribuição das espécies ao longo deste eixo possibilitou identificar duas associações de espécies (Tab. 2). A $1^{\text {a }}$ associação representou a região entre Gravatá e Laranjeiras e a $2^{\mathrm{a}}$ a llha de Porto Belo. Os agrupamentos menores descritos para o dendrograma (Fig. 4) ficaram representados ao longo do $2^{\circ}$ eixo, no qual, dentro da $1^{a}$ associação, foi possível identificar um gradiente na distribuição dos locais representado pelas estações situadas entre Laranjeiras e Gravatá, em oposição àquelas posicionadas no Rio Itajaí e sua foz (Atalaia), e dentro da $2^{\mathrm{a}}$ associação foi verificada a separação dos mesmos locais descritos para o dendrograma.

\section{DISCUSSÃO}

As associações malacológicas verificadas não apresentaram nenhuma espécie que ainda não tivesse sido registrada para a região sul brasileira (Absalão, 1987; Gonçalves e Lana, 1991; Rios, 1994, Klein et al., 2001).

Levando-se em consideração a granulometria, pode-se encontrar uma analogia com as associações descritas por Absalão (1987) e Gonçalves \& Lana (1991), na qual os gêneros (Olivancillaria, Natica, Mactra e Chione) foram similares dentro da fração dos sedimentos finos.

As coletas do presente estudo foram realizadas com o auxílio de um busca fundo, que apesar de possibilitar a estimativa da densidade, amostra uma área muito limitada, principalmente quando comparado às amostragens 
NOTAS TÉC. FACIMAR, 8: 119-126, 2004.

Tabela 1 - Identificação taxonômica dos moluscos coletados e, respectivas, freqüências absolutas e relativas (\%).

\begin{tabular}{|c|c|c|}
\hline Taxa & \multicolumn{2}{|c|}{ Freq. Abs. Freq. Rel.(\%) } \\
\hline Anadara ovalis (Bruguìere, 1789) & 1 & 8,3 \\
\hline Arcopsis adamsi (Dall, 1886) & 1 & 8,3 \\
\hline Bulla striata Bruguíere, 1792 & 1 & 8,3 \\
\hline Carditamera floridana Conrad, 1838 & 1 & 8,3 \\
\hline Chione paphia (Linnaeus, 1767) & 1 & 8,3 \\
\hline Cooperella atlantica Rehder, 1943 & 1 & 8,3 \\
\hline Corbula caribaea Orbigny, 1842 & 1 & 8,3 \\
\hline Corbula Iyoni Pilsbry, 1897 & 1 & 8,3 \\
\hline Divaricella quadrisulcata (Orbigny, 1842) & 1 & 8,3 \\
\hline Eulima sp. & 1 & 8,3 \\
\hline Mactra marplatensis Doello-Jurado, 1918 & 1 & 8,3 \\
\hline Musculus viator (Orbigny, 1846) & 1 & 8,3 \\
\hline Noethia bisulcata (Lamarck, 1819) & 1 & 8,3 \\
\hline Pholas campechiensis Gmelin, 1791 & 1 & 8,3 \\
\hline Pitar palmeri Fischer-Piette \& Testud, 1967 & 1 & 8,3 \\
\hline Semele sp. & 1 & 8,3 \\
\hline Sphenia antillensis Dall \& Simpson, 1901 & 1 & 8,3 \\
\hline Tivela mactroides (Born, 1778) & 1 & 8,3 \\
\hline Volvulella sp. & 1 & 8,3 \\
\hline Columbellidae & 1 & 8,3 \\
\hline Polyplacophora & 1 & 8,3 \\
\hline Anadara cf. brasiliana (Lamarck, 1819) & 2 & 16,7 \\
\hline Callista maculata (Linnaeus, 1758) & 2 & 16,7 \\
\hline Diplodonta danieli Klein, 1967 & 2 & 16,7 \\
\hline Dosinia concentrica (Born, 1778) & 2 & 16,7 \\
\hline Ervilia concentrica (Holmes,1860) & 2 & 16,7 \\
\hline Mactra fragilis Gmelin, 1791 & 2 & 16,7 \\
\hline Olivancillaria urceus (Roding, 1798) & 2 & 16,7 \\
\hline Olivancillaria vesica vesica (Gmelin, 1791) & 2 & 16,7 \\
\hline Semele casali Doello-Jurado, 1949 & 2 & 16,7 \\
\hline Chione subrostrata (Lamarck, 1818) & 3 & 25 \\
\hline Temnoconcha brasiliana Dall, 1921 & 3 & 25 \\
\hline Codakia costata (Orbigny, 1842) & 4 & 33,3 \\
\hline Diplodonta punctata (Say, 1822) & 4 & 33,3 \\
\hline Macoma sp. & 4 & 33,3 \\
\hline Corbula spp. & 5 & 41,7 \\
\hline Mactra iheringi Dall, 1897 & 5 & 41,7 \\
\hline Mactra isabelleana Orbigny, 1846 & 5 & 41,7 \\
\hline Nucula semiornata Orbigny, 1846 & 5 & 41,7 \\
\hline Abra sp. & 7 & 58,3 \\
\hline Natica cf. livida Pfeiffer, 1840 & 7 & 58,3 \\
\hline Tellina sp. & 10 & 83,3 \\
\hline
\end{tabular}


ALMEIDA et al:: Associações de moluscos do infralitoral de Santa Catarina.

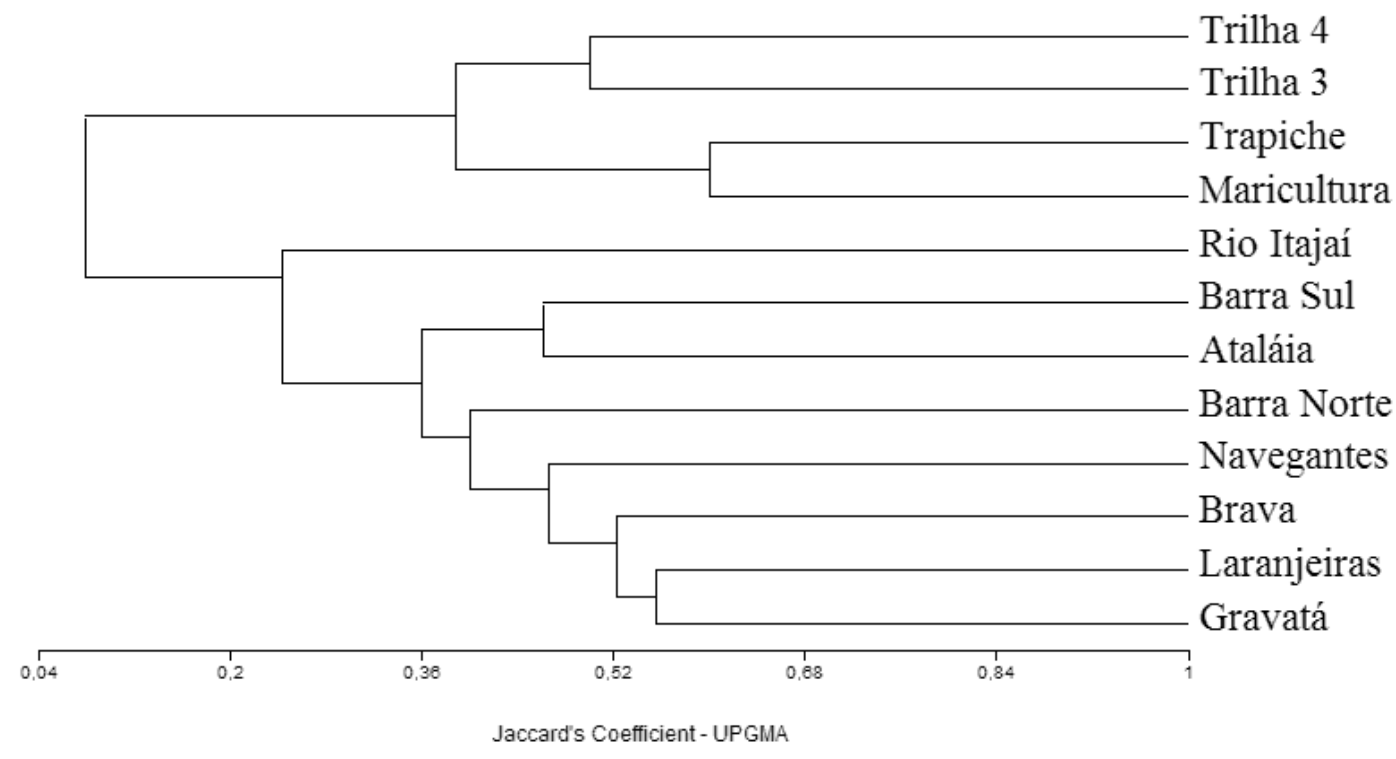

Figura 3 - Dendrograma resultante da análise de agrupamento aplicada sobre a presença e ausência dos moluscos nos locais estudados. Similaridade malacológica calculada por meio do coeficiente de Jaccard e grupos formados por meio do método das médias aritméticas não ponderadas.

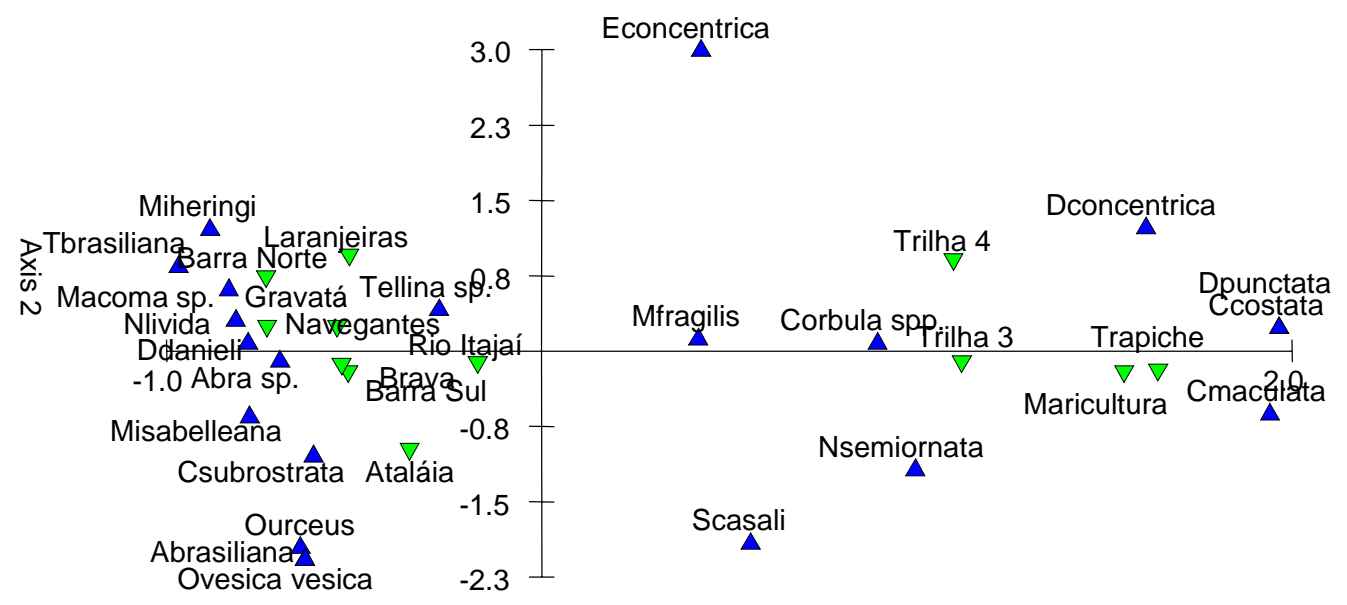

Axis 1

Figura 4 - Representação dos eixos 1 e 2 extraídos a partir da análise de correspondência sobre a presença e ausência das espécies com freqüência superior a 10\%. ( $\nabla$ representam locais e $\triangle$ espécies). 
NOTAS TÉC. FACIMAR, 8: 119-126, 2004.

Tabela 2 - Associações de espécies identificadas a partir da ocorrência nos locais amostrados ao longo do infralitoral centro norte de Santa Catarina.

\begin{tabular}{c|c}
\hline \hline Associação 1 & Associação 2 \\
\hline $\begin{array}{c}\text { Gravatá Navegantes Ataláia Brava } \\
\text { Barra Norte Barra Sul Laranjeiras }\end{array}$ & Trilha 4 Trilha 3 Maricultura Trapiche \\
\hline Temnoconcha brasiliana & Dosinia concentrica \\
Mactra iheringi & Callista maculata \\
Macoma sp. & Codakia costata \\
Natica cf. livida & Diplodonta punctata \\
Diplodonta danieli & \\
Mactra isabelleana & \\
Abra sp. & \\
Anadara cf. brasiliana & \\
Olivancillaria urceus & \\
Olivancillaria vesica & \\
Chione subrostrata & \\
\hline \hline
\end{tabular}

realizadas com dragas de arrasto. A metodologia amostral associada à faixa batimétrica amostrada neste estudo limitou as comparações com os padrões de distribuição e com as associações descritas por Absalão (1987), Gonçalves \& Lana (1991) e Klein et al. (2001), de um modo geral estes autores não amostraram em profundidades inferiores a 20 metros.

Considerando as espécies que caracterizaram a associação das estações posicionadas entre Gravatá e Laranjeiras, estas foram na grande maioria constituídas por moluscos detritívoros ou predadores, freqüentemente associados a sedimentos finos. Já aquelas que caracterizaram a llha de Porto Belo foram na totalidade constituídas por animais filtradores relacionados a sedimentos mais arenosos.

Padrão similar foi encontrado no trabaIho de Gonçalves e Lana op. cit., ao longo da plataforma continental, mostrando a relação entre a distribuição dos moluscos, a granulometria do sedimento e seus hábitos alimentares.
O maior número de espécies na $1^{\text {a }}$ associação pode estar relacionado à maior abrangência da área amostrada (Gravatá a Laranjeiras), enquanto que a área da $2^{\mathrm{a}}$ associação restringiu-se ao entorno da llha de Porto Belo.

Por fim, destaca-se que das 4 espécies que integraram a associação da llha, duas podem atingir até mais que $50 \mathrm{~mm}$ de comprimento (Callista maculata e Dosinia concêntrica) e poderiam ser cultivadas, assim como outros venerídeos, pectinideos, ostreideos, mactrideos e donacideos, conforme mencionado por Lana (1996) e Gaspar et al. (2004).

\section{REFERÊNCIA BIBLIOGRÁFICA}

Absalão, R. S., 1986, Discriminação Ambiental entre Associações de Moluscos MacroBentônicos ao Largo de Rio Grande, RS, Brasil. Situação Inverno-Primavera. MSc. Thesis. Universidade do Rio Grande, RS, 120p. 
Absalao, R. S. 1987. Associacoes Malacologicas Ao Largo de Rio Grande, Rs. As Comunidades Paralelas de Thorson e As Associacoes Benticas de Peres. Anais do Simpósio sobre Ecossistemas da Costa Sul e Sudeste Brasileira. 2:401 - 414.

Absalão, R.S. 1989. Padrões distributivos e zoogeografia dos moluscos da plataforma continental brasileira. Parte III. Comissão oceanográfica Espírito Santo I. Mem. Inst. Oswaldo Cruz. 8(4):1-6.

Absalão, R.S. \& R.L. Cruz. 1990. Moluscos da plataforma continental brasileira. Parte II: Comissão Oceanográfica "GEOMAR X". Naturalia. 15:21-33.

Arruda, E.P.de. \& A.C.Z. Amaral. 2003. Spatial distribution of mollusks in the intertidal zone of sheltered beaches in southeastern of Brazil. Rev. Bras. Zool. 20(2):291-300.

Folk, R. L. \& W.C. Ward. 1957. Brazos River bar: a study in the significance of grain size parameters. J. Sedim. Petrol. 27: 3-26.

Gaspar, M.; Sobral, M.; Maia, F.; Sobral, M.P.; Viegas, M.C. \& C.C. Monteiro. 2004. A pescaria de Moluscos Bivalves na Zona Ocidental Norte e Zona Sul. Ponto de Situação dos Princiapais Bancos (Setembro/ Outubro 2002). Relat. Cient. Téc. IPIMAR, Série digital, № 12, 33p., il.

Gonçalves, E.M. \& P.C. Lana. 1991. Padrões de distritbuição de bivalvia e gastropoda da plataforma continental da costa sudeste do Brasil $\left(24^{\circ} \mathrm{S}-27^{\circ} \mathrm{S}\right)$. Nerítica. 6:73-92.
Klein, J. A.; Borzone, C. A. \& P.R. Pezzuto. 2001. A macro e megafauna bêntica associada aos bancos de Vieira Euvola zivzac (mollusca: bivalvia) no Litoral Sul do Brasil. Atlântica. 23:17-26.

Lana, P.C., Camargo, M.G., Brogim, R.A \& V. Isaac. 1996. O bentos da costa brasileira. Avaliação crítica e levantamento bibliográfico. Ministério do Meio Ambiente, dos Recursos hídricos e da Amazônia Legal/ Comissão Interministerial para os Recursos do Mar/Fundação de Estudos do Mar, Rio de Janeiro, $431 \mathrm{pp}$.

Legendre, L. \& P. Legendre. 1998. Numerical ecology. developments in environmental modelling, 3. Elsevier Sci. Publ. Company, 419p.

Migotto, A.E.; Tiago, C.G. \& A.R.M. Magalhães, 1993. Malacofauna marinha da região costeira do Canal de São Sebastião, SP, Brasil: Gastropoda, Bivalvia, Polyplacophora e Scaphopoda. Bolm. Inst. Oceanogr. 41:1327.

Rios, E. 1994. Sheashells of Brazil. 2 ed. Rio Grande: FURG. 368pp.

Schettini, C.A.F. \& J.L.B. Carvalho. 1998. Hidrodinâmica e distribuição de sólidos em suspensão no estuário do Rio Itajaí-Açu. (Relatório). Notas Téc. Facimar. 2:131-140. Suguio, K. 1979. Introdução a Sedimentologia. Blücher Ed. Universidade de São Paulo. 312pp. 